\title{
Differential dynamic microscopy microrheology of soft materials: A tracking-free determination of the frequency-dependent loss and storage moduli
}

\author{
Paolo Edera, ${ }^{1}$ Davide Bergamini, ${ }^{1}$ Véronique Trappe,${ }^{2}$ Fabio Giavazzi, ${ }^{1, *}$ and Roberto Cerbino ${ }^{1, \dagger}$ \\ ${ }^{1}$ Dipartimento di Biotecnologie Mediche e Medicina Traslazionale, Università degli Studi di Milano, \\ Via. F.lli Cervi 93, Segrate (MI) I-20090, Italy \\ ${ }^{2}$ Department of Physics, University of Fribourg, Chemin du Musée 3, CH-1700, Fribourg, Switzerland
}

(Received 3 August 2017; published 29 December 2017)

\begin{abstract}
Particle-tracking microrheology (PT- $\mu \mathrm{r}$ ) exploits the thermal motion of embedded particles to probe the local mechanical properties of soft materials. Despite its appealing conceptual simplicity, PT- $\mu$ r requires calibration procedures and operating assumptions that constitute a practical barrier to its wider application. Here we demonstrate differential dynamic microscopy microrheology (DDM- $\mu \mathrm{r}$ ), a tracking-free approach based on the multiscale, temporal correlation study of the image intensity fluctuations that are observed in microscopy experiments as a consequence of the translational and rotational motion of the tracers. We show that the mechanical moduli of an arbitrary sample are determined correctly over a wide frequency range provided that the standard DDM analysis is reinforced with an iterative, self-consistent procedure that fully exploits the multiscale information made available by DDM. Our approach to DDM- $\mu \mathrm{r}$ does not require any prior calibration, is in agreement with both traditional rheology and diffusing wave spectroscopy microrheology, and works in conditions where PT- $\mu$ r fails, providing thus an operationally simple, calibration-free probe of soft materials.
\end{abstract}

DOI: 10.1103/PhysRevMaterials.1.073804

\section{INTRODUCTION}

Rheology is a well-established experimental technique that probes the response of materials upon application of a stress or strain [1]. This probe is particularly significant for soft materials such as paint, starch, mayonnaise and gelatin that defy the sharp rules according to which we tend to classify a substance as an ideal viscous Newtonian liquid or a perfectly elastic Hookean solid. In practice, depending on the timescales probed and the magnitude of the stress or strain applied, soft materials may behave as solids or liquids. For instance, a dense colloidal suspension may exhibit a solid-like response upon application of small-amplitude, fast deformations whereas it may be more similar to a liquid upon applying largeamplitude, slow deformations. Converting these important but qualitative considerations into some quantitatively and reproducibly determined mechanical moduli of the materials is the realm of rheology [2].

Traditional rheology makes use of rheometers, in which a soft material is loaded into the gap between two solid surfaces and stressed (or strained) in a controlled fashion to measure the strain (or stress) response of the material. This response can be entirely ${ }^{1}$ described in terms of a complex modulus $G^{*}(\omega)=$ $G^{\prime}(\omega)+i G^{\prime \prime}(\omega)$. $G^{*}$ can be measured with a rheometer, by imposing, for instance, an oscillatory strain $\gamma(t)=\gamma_{0} \sin (\omega t)$ and measuring the stress $\sigma(t)$ developed by the material. In general, one finds that $\sigma(t)=G^{\prime} \gamma_{0} \sin (\omega t)+G^{\prime \prime} \gamma_{0} \cos (\omega t)$, where $G^{\prime}$ and $G^{\prime \prime}$ are the storage (or elastic) and loss (or viscous) moduli of the material, respectively.

This denomination denotes that a Hookean solid is characterized only by a stress in phase with the applied strain,

\footnotetext{
*fabio.giavazzi@unimi.it

†roberto.cerbino@unimi.it

${ }^{1}$ As long as the stress or strain is small enough not to change materials' mechanical properties.
}

with $G^{\prime}$ corresponding to the elastic modulus of the solid, whereas the response of a Newtonian liquid is in quadrature, with $G^{\prime \prime}=\eta \omega$, where $\eta$ is the dynamic viscosity. A generic soft material will have an in-phase response that can be associated with its solid-like character and an in-quadrature response that is due to its liquid-like nature. Naively one can say that if for a given frequency $G^{\prime} \gg G^{\prime \prime}$ the material is substantially a solid, whereas if $G^{\prime} \ll G^{\prime \prime}$ it behaves as a liquid. Inspecting the full frequency dependence of $G^{\prime}$ and $G^{\prime \prime}$ provides thus a fundamental tool to classify materials based on their mechanical response or to monitor changes in their mechanical properties during, for instance, gelation or aggregation processes [3].

Despite their power and immediacy, rheology tests performed with a rheometer are affected by some limitations: they require a large quantity of material (on the order of a few milliliters), they average-over possible heterogeneities of the sample, and the accessible frequency range is limited at small $\omega$ by torque limitations and at large $\omega$ by inertial effects [4].

A complementary approach that addresses the above issues is represented by microrheology [5-9]. Originally introduced by Mason and Weitz in 1995 [10], the so-called passive microrheology consists of seeding the soft material of interest with tracer particles of radius $a$ and measuring the mean-square displacement (MSD) $\left\langle\Delta r^{2}(t)\right\rangle$ of the tracers within the material as a function of time $t$. If the material is homogeneous on the length scale of the tracers size, the MSD of noninteracting tracers can be related to the frequency-dependent complex modulus $G^{*}(\omega)$ by using the generalized Stokes-Einstein relation (GSER) [7]

$$
G^{*}(\omega)=\left.\frac{d k_{B} T}{3 \pi a s\left\langle\Delta \tilde{r}^{2}(s)\right\rangle}\right|_{s=i \omega},
$$

where $d$ is the number of dimensions tracked in the MSD, $k_{B}$ is the Boltzmann constant, $T$ is the temperature, $i$ is the imaginary unit, and $\left\langle\Delta \tilde{r}^{2}(s)\right\rangle$ is the Laplace transform of the 
MSD. In a Newtonian liquid of viscosity $\eta$, the MSD of a tracer particle with diffusion coefficient $D_{0}=k_{B} T /(6 \pi \eta a)$ is given by $\left\langle\Delta r^{2}(t)\right\rangle=2 d D_{0} t$, which leads to $\left\langle\Delta \tilde{r}^{2}(s)\right\rangle=2 d D_{0} / s^{2}$ and, in turn, to $G^{*}(\omega)=i G^{\prime \prime}(\omega)=i \omega \eta$. For a solid, instead, the elastic modulus $G^{*}(\omega)=G^{\prime}$ is obtained from estimating the mean-square displacement $\left\langle\Delta r^{2}(t)\right\rangle=\frac{d k_{B} T}{3 \pi a G^{\prime}}$ of a particle in an elastic trap with the condition $\left\langle\Delta r^{2}(t)\right\rangle=0$ for $t<0$. The Laplace transform is then given by $\left\langle\Delta \tilde{r}^{2}(s)\right\rangle=\frac{d k_{B} T}{3 \pi a G^{\prime}} \frac{1}{s}$.

The MSD of the tracer particles can be obtained in a variety of ways $[5,7,8]$. A direct way is to track in real space the trajectories of the tracer particles, as done in particle-tracking (PT) experiments [6]. An alternative way is to extract the MSD from the measurement of the intensity scattered or fluorescently emitted by a dilute collection of noninteracting tracer particles, as done in diffusing wave spectroscopy (DWS) [10], dynamic light scattering (DLS) $[11,12]$, and fluorescence correlation spectroscopy (FCS) [13]. Historically, DWS-microrheology (DWS- $\mu \mathrm{r}$ ) was the first to be developed and, together with PT- $\mu \mathrm{r}$, is still one of the most common approaches. DWS and PT are in principle quite complementary: DWS gives access to short timescales and small MSD, while PT gives access to longer timescales and larger MSD. However, while DWS can be used with almost no user intervention, PT involves a rather tedious and delicate selection of the trajectories. The disadvantage of DWS in turn is to require larger tracer-particle concentrations that may more easily alter the mechanical properties of the material itself.

An alternative realization of passive microrheology was introduced in 2003 by Cheng and Mason [14] under the name of rotational diffusion microrheology. This approach exploits the thermal fluctuations associated with the rotational, rather than translational, degrees of freedom of immersed tracer particles to infer the viscoelastic properties of the host fluid. In fact, under the same hypotheses of Eq. (1), the following rotational GSER holds [14]:

$$
G^{*}(\omega)=\left.\frac{k_{B} T}{4 \pi a^{3} s\left\langle\Delta \tilde{\theta}^{2}(s)\right\rangle}\right|_{s=i \omega},
$$

which relates the complex modulus $G^{*}(\omega)$ to the Laplace transform of the angular mean-square displacement (AMSD) of the tracer $\left\langle\Delta \tilde{\theta}^{2}(s)\right\rangle$ around a given axis. In general, the measurement with optical means of the rotational dynamics is technically more challenging than its translational counterpart and usually requires the use of specialized shape [14-16] or optically [17-20] anisotropic tracers. Rotational diffusion microrheology has been initially demonstrated by using a light streak tracking method enabling the time-resolved measurement of the axial orientation of a single, optically trapped microdisk [14]. Other experimental realizations were based on depolarized DLS $[17,18]$ and, by using suitably large (micron-sized) anisotropic tracers, on video PT [15].

Almost ten years ago, the usefulness of a technique named differential dynamic microscopy (DDM) was demonstrated for the characterization of the translational dynamics of colloidal suspensions of particles [21]. One of the main features of DDM is that it lies somehow between PT and DLS. Similar to PT, it is based on real-space movies collected in microscopy experiments. These data are treated via an image processing algorithm [22] or equivalent versions of it [23] that combines image differences and spatial Fourier transformations to obtain as a result the intermediate scattering function $f(\mathbf{q}, t)$ that is typically probed in DLS experiments as a function of the scattering wave vector $\mathbf{q}$ and time $t$ [24]. Since its introduction, DDM has been profitably used and also extended by several groups [25-34] for a variety of applications [35]. In particular, DDM has been recently proven to be an effective tool to measure also the rotational dynamics of anisotropic colloidal particles in solution. This can be accomplished, for example, by introducing in the microscope set-up suitably oriented polarizing elements (polarized DDM or p-DDM [20]) or by adopting a dark-field imaging condition (dark-field DDM or DF-DDM [16,36]).

In this work, we show that DDM can indeed be used as a convenient and reliable tool to probe the mechanical properties of complex fluids, which we demonstrate with both Newtonian liquids obtained by mixing water and glycerol in variable proportions, and viscoelastic samples consisting of aqueous solutions of a high-molecular-weight polymer (polyethylene oxide). Compared to very recent experiments [37], where DDM- $\mu r$ was demonstrated with a classical DDM analysis, we determine here the tracer MSD with a fitting-free, optimization-based procedure that is applicable to an arbitrary sample and does not require any prior calibration. The obtained results are found to be in agreement with standard rheology and with both PT- $\mu$ r and DWS- $\mu$ r. In addition, we show that DDM- $\mu$ r succeeds in probing the rotational degrees of freedom of the embedded particles and that it operates also with small tracer particles that are not suitable for tracking experiments; this widens the range of applicability of microrheology.

Our results show that optimization-based DDM- $\mu \mathrm{r}$ is a flexible, calibration-free approach to microrheology that can be almost fully automated, thus eliminating the arbitrariness, typical of PT experiments, in sorting and selecting the suitable trajectories. We expect that DDM- $\mu$ r can be successfully used to measure the rheological properties of a variety of soft materials, also in cases where DLS, DWS, and PT cannot be used. A typical example is the cell interior [38] where DDM has already been successfully used to measure the interplay of diffusion and flow during oogenesis.

\section{MATERIALS AND METHODS}

\section{A. Sample preparation}

We used two different classes of samples: Newtonian fluids with varying viscosity obtained by adding different amounts of glycerol to water, and viscoelastic fluids consisting of aqueous solutions of polyethylene oxide (PEO, $\left.M_{W}=2 \times 10^{6} \mathrm{Da}\right)$. For the PEO solution we chose to work at a concentration of $c=2.0 \mathrm{wt} \%$ above the overlap concentration $\left(c^{*}=0.09 \mathrm{wt} \%\right)$ to obtain a sample with appreciable viscoelastic properties in the frequency range of interest. At these conditions, the mesh size of the polymer network is estimated to be $\sim 10 \mathrm{~nm}[12]$.

\section{Glycerol-water solutions}

The glycerol-water samples were prepared by mixing suitable amounts of glycerol (Sigma Aldrich), MilliQ water, and an aqueous suspension of latex beads (Sigma Aldrich, LB5, nominal diameter $0.45-0.47 \mu \mathrm{m}$, solid content $10 \%$ ) to 
reach final mass fractions of glycerol equal to $0 \%, 48.8 \%$, $82.7 \%$, and $97.5 \%$. The mass fractions of suspended beads were $0.075 \%, 0.14 \%, 0.18 \%$, and $0.2 \%$, respectively. All four samples were investigated by using both PT and DDM.

\section{Polymer solution}

The PEO solution for traditional rheology experiments was prepared from the pure product purchased as powder (Sigma Aldrich, prod. code 372803). The powder was carefully dissolved in MilliQ water previously filtered with membrane filters (pore size $0.2 \mu \mathrm{m}$ ). To prevent the formation of clumps of undissolved polymer, water was gradually added to the polymer while stirring. The solution was then kept in incubation for nine days at about $40{ }^{\circ} \mathrm{C}$. A few drops of a sodium azide solution (molarity $4 \mathrm{mM}$ ) were added to the PEO solution to prevent bacterial proliferation.

For the DDM, PT, and DWS experiments, in which colloidal tracer particles were to be added to the PEO solution, a prescribed amount of pure water was replaced with the aqueous colloidal suspensions of latex beads during sample preparation. The beads were purchased from Sigma Aldrich with the part numbers LB1 (nominal diameter 0.10-0.12 $\mu \mathrm{m}$, solid content 10\%) and LB5 (nominal diameter 0.45-0.47 $\mu \mathrm{m}$, solid content $10 \%$ ). The bead sizes were also tested with DDM and were found to be equal to $0.112 \pm 0.002 \mu \mathrm{m}$ and $0.445 \pm 0.005 \mu \mathrm{m}$ for the LB1 and LB5 samples, respectively. After dilution the final concentration of PEO was $2 \% \mathrm{wt} / \mathrm{wt}$ and the final concentration of tracer beads was $(1.00 \pm 0.05) \times$ $10^{-3} \mathrm{wt} / \mathrm{wt}$. To ensure multiple scattering for the DWS experiment we used LB5 particles at $(1.00 \pm 0.05) \times 10^{-2}$ wt/wt.

For the p-DDM experiment, a small amount of optically anisotropic tracer was added to the PEO solution. The tracer used in this study consists of spherical colloidal particles, with a polymerized nematic liquid-crystal core, whose birefringence makes the particles optically anisotropic, with a uniaxial symmetry $[18,19,39,40]$. The particles were prepared and purified according to the protocols described in detail in Refs. [39,41]. The final mass fraction of the dispersion was approximately 4\%. After dilution, the final concentration of PEO was $2 \% \mathrm{wt} / \mathrm{wt}$, while the concentration of optically anisotropic tracers was $(1.8 \pm 0.2) \times 10^{-3} \mathrm{wt} / \mathrm{wt}$. The diameter of the tracers $(0.22 \pm 0.03 \mu \mathrm{m})$ was determined by measuring with bright-field DDM the translational diffusion coefficient of a diluted dispersion in pure water.

\section{B. Rheology}

Since for the Newtonian glycerol-water mixtures, reliable literature data are available, standard rheology experiments were only performed for the PEO sample. We used a commercial rheometer (Anton Paar MCR502) equipped with cone and plate geometry (radius $=25 \mathrm{~mm}$, cone angle $=1^{\circ}$ ) to apply an oscillatory shear strain with strain amplitude of $5 \%$, and angular frequency in the range $[0.1,100] \mathrm{rad} \mathrm{s}^{-1}$. Our experiments were performed in the temperature range $T=20-25^{\circ} \mathrm{C}$. To avoid evaporation during measurement we used a solvent trap.

\section{Particle tracking}

PT experiments were performed by tracking LB5 particles dispersed in the four glycerol-water samples and in a polymer solution with the PEO concentration also used in Sec. II B. The samples were loaded in a capillary (Vitrocom) with rectangular cross section and internal dimensions $10 \times 2 \times 0.1 \mathrm{~mm}^{3}$. Microscopy experiments were performed in bright field with an optical microscope (Nikon Ti-E) equipped with a digital camera (Hamamatsu Orca Flash 4.0 v2), and a $40 \times$ objective. The resulting pixel size was $d_{\text {pix }}=162.5 \mathrm{~nm}$. Image sequences made of 10000 images $(512 \times 256$ pixels $)$ were acquired at two different frame rates $(777$ and $10 \mathrm{~Hz})$. In all acquired images, the sample appeared transparent and the colloidal particles were clearly visible.

Particle-tracking analysis was conducted by using a customized version of the MATLAB code script made freely available by the Kilfoil group at the University of Massachusetts [42]. This software reconstructs the individual trajectories of several particles in parallel, calculating their MSD as a function of time. Compared to the original code, we added some custom features, mainly to adapt our analysis to bright-field time-lapse movies and to estimate error bars and experimental uncertainties.

Once the MSD was obtained, the data needed to be corrected by subtracting the additive contribution due to the intrinsic localization uncertainty that becomes dominant for small times and particle displacements [43]. This step, which lies at the core of PT- $\mu$ r, requires an independent calibration of the particle localization error. In our experiments, the static localization error was determined as that which minimizes the deviation from a purely linear behavior in the MSD measured in Newtonian samples [8].

Once the corrected MSD was obtained, we followed different procedures for the two classes of samples. Results for MSD of the Newtonian fluids were simply fit to a straight line and the sample viscosity $\eta$ was obtained from the slope, $4 k_{B} T /(6 \pi \eta a)$. For the PEO sample, we used the Kilfoil-group software to extract the frequency-dependent elastic and loss moduli, $G^{\prime}$ and $G^{\prime \prime}$, respectively. The software implements an algebraic inversion procedure based on the work of Mason et al. [6].

\section{Diffusing wave spectroscopy}

DWS microrheology experiments were performed on a polymer solution with the PEO concentration also used in Sec. II B. The tracer particles concentration (1\%) was chosen to ensure multiple scattering [10]. In the limit of multiple scattering the autocorrelation function of the scattering intensity is given by

$$
g(\tau)=\int_{0}^{\infty} P(s) e^{-\frac{k_{0}^{2}}{3}\left\langle\Delta r^{2}(\tau)\right\rangle \frac{s}{l^{*}}} d s,
$$

where $k_{0}=2 \pi n / \lambda_{0}$ is the wave vector of light with wavelength $\lambda_{0}$ (in our experiment $687 \mathrm{~nm}$ ) incident on a medium with refractive index $n . P(s)$ is the scattering-geometrydependent relative probability distribution of photon path lengths $s$ inside the medium and $l^{*}$ is the transport mean free path, which quantifies the distance that a photon has to travel inside the sample before loosing memory of its original 


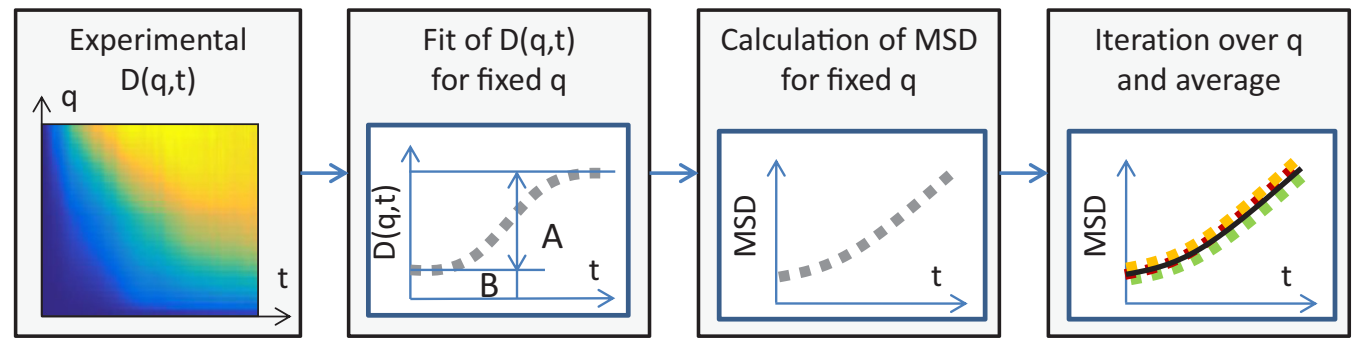

FIG. 1. Schematic representation of the fitting-based procedure used to determine MSD from the image structure function. For a given wave vector $q$, the image structure function $D(q, t)$ is fit to a model to obtain the noise baseline $B(q)$ and the signal amplitude $A(q)$. Using Eq. (7), an estimate for the MSD is obtained. The procedure is then repeated for all $q$ values in the selected $q$ range and the best estimate for the MSD is obtained as the average of the curves obtained for all $q$ values.

direction. For our sample, we found $l^{*}=256 \pm 5 \mu \mathrm{m}$. The MSD can be extracted by inverting Eq. (3) [44].

For our experiments we used the commercial instrument DWS Rheolab (LSInstruments, Fribourg, Switzerland), a compact stand-alone optical microrheometer that is based on DWS. The sample was hosted in a cuvette of thickness $L=2 \mathrm{~mm}$. Measurements were performed in transmission geometry with a duration of $3000 \mathrm{~s}$ each. The MSD of the tracer particles was obtained in the time range $1.38 \mu$ s to $0.71 \mathrm{~s}$ and subsequently analyzed to extract the moduli $G^{\prime}$ and $G^{\prime \prime}$.

\section{E. Differential dynamic microscopy}

We performed DDM measurements on all samples. LB5 particles were used for the four Newtonian samples and for the PEO solution. The latter was also studied with LB1 particles; for this sample PT is not feasible. Standard DDM analysis was based on a repeated sequence of image subtractions and image Fourier transforms [21,23,24]. In more detail, the image structure function for all the accessible two-dimensional wave vectors $\mathbf{q}=\left(q_{x}, q_{y}\right)$ was calculated for a set of time delays $t$ according to

$$
D(\mathbf{q}, t)=\left\langle\left|I\left(\mathbf{q}, t_{0}+t\right)-I\left(\mathbf{q}, t_{0}\right)\right|^{2}\right\rangle_{t_{0}},
$$

where $I(\mathbf{q}, t)$ is the Fourier transform of the image $I(\mathbf{x}, t)$ acquired at time $t$ in a fixed plane in which the horizontal position is labeled by $\mathbf{x}=(x, y)$. It has been recently shown that multiplying the images with a windowing function before performing the Fourier transform operation removes the artifacts due to the finite image size and improves the determination of $D(\mathbf{q}, t)$, especially for those $q$ for which the signal is comparable or smaller than the noise [46]. We thus apply this algorithm in our analysis.

The image structure function is quantitatively related to the normalized (translational) intermediate scattering function $f_{T}(\mathbf{q}, t)$ and in most cases of interest the simple relationship

$$
D(\mathbf{q}, t)=A(\mathbf{q})\left[1-f_{T}(\mathbf{q}, t)\right]+B(\mathbf{q})
$$

holds, where the functions $A(\mathbf{q})$ and $\boldsymbol{B}(\mathbf{q})$, usually treated as fitting parameters, are set by the spatial intensity correlations and the noise of the detection chain, respectively. The normalized intermediate scattering function has some general properties such that $f_{T}(\mathbf{q}, 0)=1$ and $f_{T}(\mathbf{q}, t \rightarrow \infty)=0$ if the particles position are fully uncorrelated for long times [47]. For noninteracting particles in a homogeneous medium, the probability distribution of particle displacements is Gaussian and as a consequence one has [47]

$$
f_{T}(\mathbf{q}, t)=e^{-\frac{q^{2}}{4}\left\langle\Delta r^{2}(t)\right\rangle},
$$

which is the two-dimensional equivalent of the main assumption on which DLS microrheology is based $[6,12]$. Thus, under conditions in which Eq. (6) holds, the MSD of tracer particles dispersed in a soft material can be obtained as

$$
\left\langle\Delta r^{2}(t)\right\rangle=-\frac{4}{q^{2}} \ln \left(1-\frac{D(\mathbf{q}, t)-B(\mathbf{q})}{A(\mathbf{q})}\right),
$$

provided that an accurate fitting of the structure functions can be performed, as sketched in Fig. 1. Once the MSD is obtained it can be used to estimate the loss and elastic moduli of the sample, which is done here with the same tools used to treat PT data.

We note that, even though the whole procedure to extract the MSD from the DDM analysis of microscope movies appears at first rather straightforward, a successful and accurate output requires the precise knowledge of $A(\mathbf{q})$ and $B(\mathbf{q})$. When an accurate fitting model for $\left\langle\Delta r^{2}(t)\right\rangle$ is available, as in the case of freely diffusing particles where $\left\langle\Delta r^{2}(t)\right\rangle=4 D_{0} t$, this can be done also if the key experimental parameters (image exposure time, acquisition frame rate, total number of images) do not allow us to observe the full relaxation of the intermediate scattering function from one to zero. By contrast, if such a model is not available, any spurious effect altering the determination of $A$ and $B$ will impair the determination of a correct MSD. Clearly, if DDM is to be used as a general-purpose probe of the mechanical properties of soft materials, suitable precautions need to be taken to guarantee a model-free determination of the MSD. In Sec. III B, we show how this task can be accomplished by replacing the standard fitting-based DDM analysis with a suitable optimization-based DDM analysis.

\section{F. Polarized differential dynamic microscopy}

p-DDM measurements were performed in combination with standard bright-field DDM on the PEO solution. p-DDM is a recently introduced extension of DDM allowing the simultaneous measurement of the rotational and translational dynamics of dispersed optically anisotropic colloidal particles observed between suitably oriented polarizing elements [20].

In $\mathrm{p}$-DDM, the image structure function $D_{p}(\mathbf{q}, t)$ is determined with the same procedure described in the previous 
section but, at a variance with standard DDM, it also provides information on the rotational dynamics of the particles. In particular, instead of Eq. (5) we have

$$
D_{p}(\mathbf{q}, t)=A_{p}(\mathbf{q})\left[1-f_{p}(\mathbf{q}, t)\right]+B_{p}(\mathbf{q}) .
$$

The intermediate scattering function $f_{p}$ shows in this case two distinct decays: a faster one associated with the rototranslational dynamics and a slower one associated with the translational dynamics alone:

$$
f_{p}(\mathbf{q}, t)=\alpha(\mathbf{q}) f_{R T}(\mathbf{q}, t)+[1-\alpha(\mathbf{q})] f_{T}(\mathbf{q}, t) .
$$

Here, $f_{T}(\mathbf{q}, t)$ is the translational intermediate scattering function given in Eq. (6), while $f_{R}(t)=e^{-3\left\langle\Delta \theta^{2}(t)\right\rangle}$ and $f_{R T}(\mathbf{q}, t)=f_{R}(t) f_{T}(\mathbf{q}, t)$ are the rotational and the rototranslational intermediate scattering functions, respectively $[17,20]$. We note that the translational intermediate scattering function $f_{T}$ coincides with the full intermediate scattering function obtained in bright-field experiments [see Eq. (6)]. $\alpha(\mathbf{q})$ is a weighting factor determined by the optical properties of the particles and by the angle between the polarizing elements [20]. As described in detail in Appendix B, by combining p-DDM and bright-field DDM measurements, we were able to remove the purely translational contributions from the image structure function, obtaining an estimate of the rotational intermediate scattering function $f_{R}(t)$, from which AMSD is extracted as

$$
\left\langle\Delta \theta^{2}(t)\right\rangle=-\frac{1}{3} \ln f_{R}(t) .
$$

The AMSD is then used to estimate the viscoelastic moduli of the fluid, with the same procedure used to treat PT and standard DDM data.

The sample was loaded in a glass capillary as described in Sec. II C. Microscopy experiments were performed both in bright field (standard DDM) and by sandwiching the sample between two polarizers that are mutually oriented at an angle $\beta=75^{\circ}$ ( $\mathrm{p}$-DDM configuration). To ensure approximately the same transmitted intensity in both configurations, a neutral density filter of optical density 2 was inserted in the optical path during bright-field measurements. With the exception of the alternative presence of the neutral density filter and of the polarizers, microscope and camera settings were kept unchanged for all the measurements. We used the microscope and the camera described in Sec. II C, in combination with a $20 \times$ objective. After $2 \times$ binning, the effective pixel size was $d_{\text {pix }}=650 \mathrm{~nm}$. Image sequences made of 50000 images $(256 \times 128$ pixels $)$ were acquired at two different frame rates (777 and $2 \mathrm{~Hz}$ ).

\section{RESULTS AND DISCUSSION}

\section{A. Newtonian fluids: Fitting-based differential dynamic microscopy analysis}

The DDM experiments presented here are aimed at measuring the viscosity of the Newtonian fluids seeded with $\simeq 0.5 \mu \mathrm{m}$ latex beads (LB5) described in detail in Sec. II. The key step of the analysis consists of extracting the MSD of the tracers directly from the image structure function by using Eq. (7). To this aim the amplitude $A(\mathbf{q})$ and the baseline $B(\mathbf{q})$ need to be known with high accuracy for each $q$,
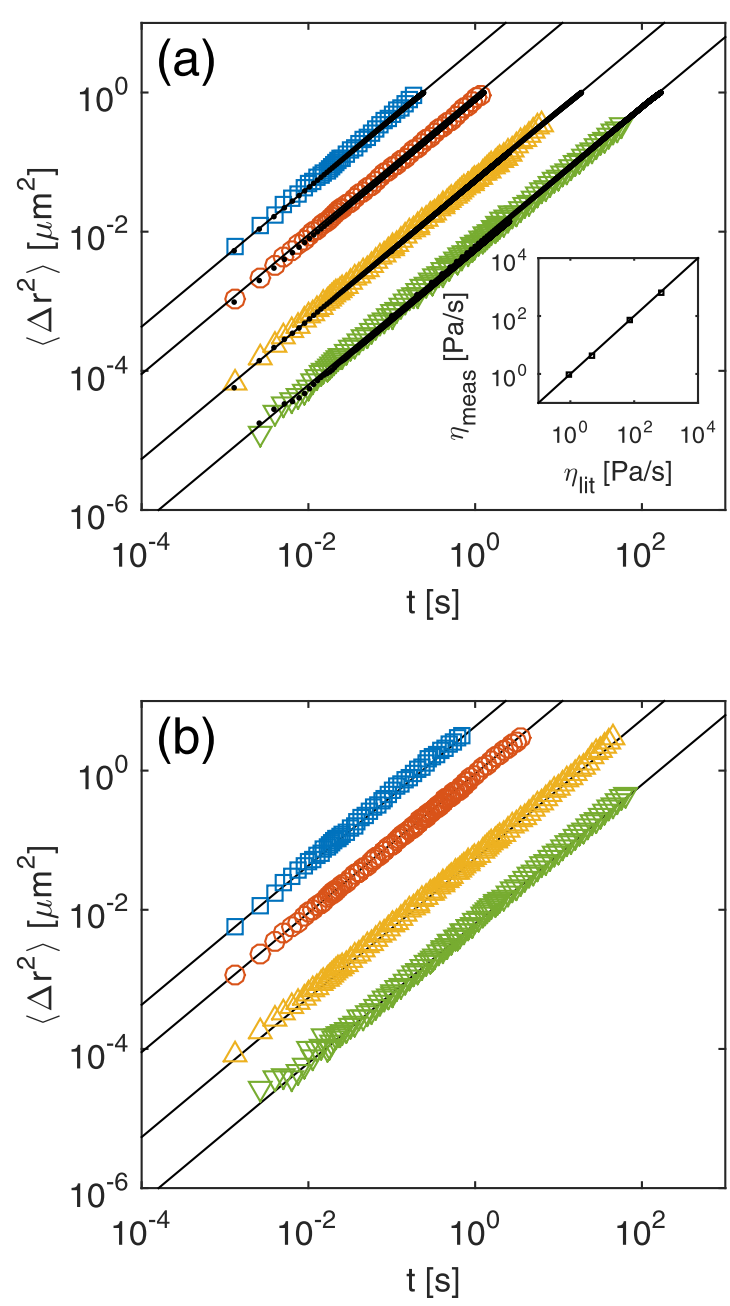

FIG. 2. MSD of LB5 tracers in different water-glycerol solutions. (a) Symbols: MSD obtained from DDM with the fitting-based procedure (details in main text); dots: MSD obtained from PT; continuous lines: expected MSD from Ref. [45]. The viscosities $\eta_{\text {meas }}$ experimentally determined with DDM are shown in the inset as a function of the expected values $\eta_{\text {lit }}$ (symbols). The error bars are smaller than the symbols and the continuous line corresponds to the identity $\eta_{\text {meas }}=\eta_{\text {lit }}$. (b) Symbols: MSD obtained from DDM with the model-free procedure (see main text for details); continuous lines: expected MSD, as in panel (a).

because any systematic error in their determination would introduce a bias in the MSD. In particular, an overestimate (underestimate) of $B(\mathbf{q})$ would lead to a spurious acceleration (deceleration) of the reconstructed tracers dynamics for small times.

For monodisperse noninteracting colloidal particles dispersed in a Newtonian fluid, the intermediate scattering function decays exponentially $f(\mathbf{q}, t)=e^{-D_{0} q^{2} t}$ and $A(\mathbf{q})$ and $B(\mathbf{q})$ can be simply obtained by fitting the image structure functions to Eq. (5).

In our experiments, we found indeed that for all samples the intermediate scattering functions were very well described in terms of a single exponential relaxation for all the wave vectors in the range [2.27,9.82] $\mu \mathrm{m}^{-1}$ for pure water, of $[2.27,9.06] \mu \mathrm{m}^{-1}$ for $48.8 \%$ glycerol in water, 


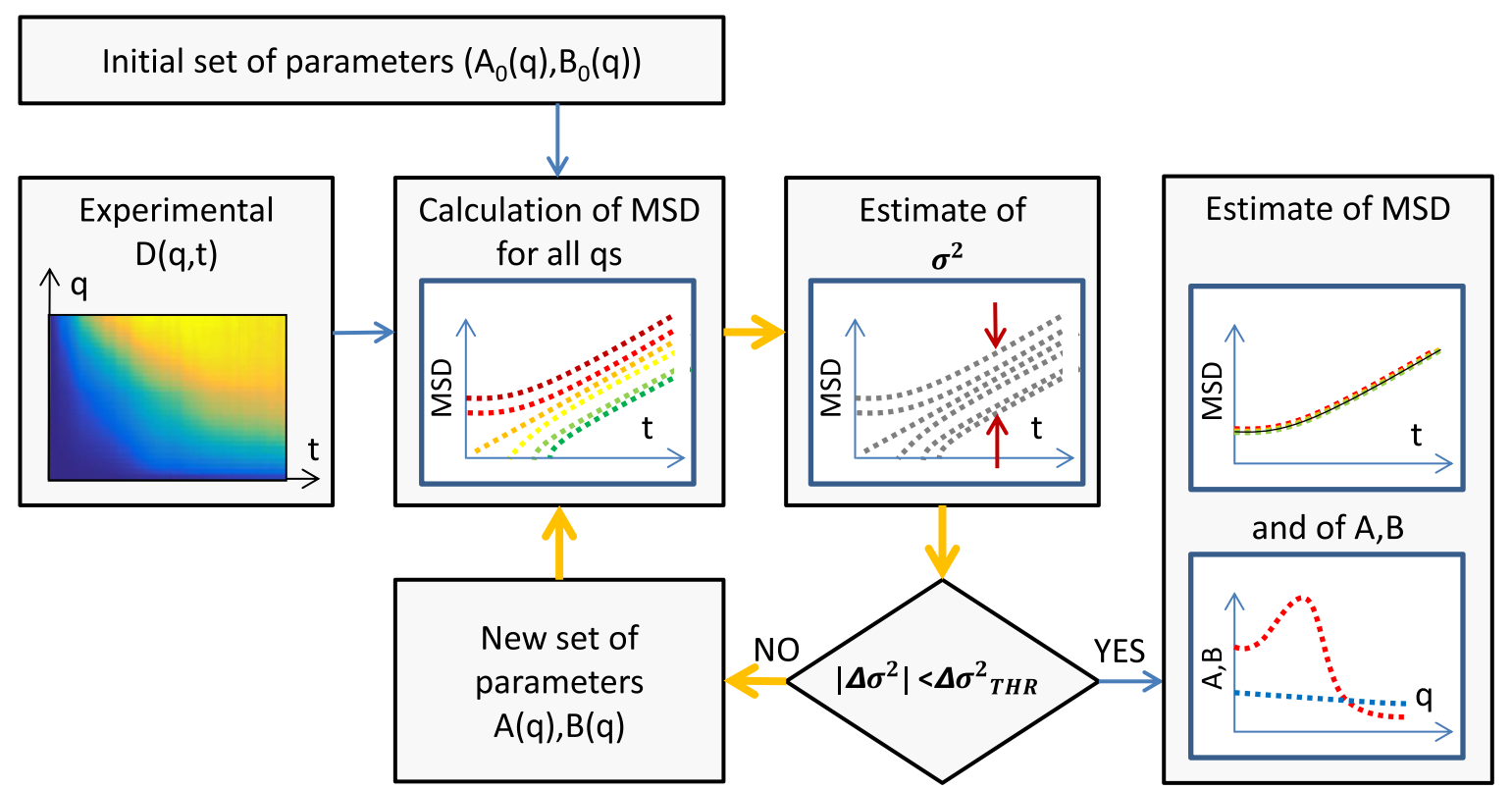

FIG. 3. Schematic representation of the optimization-based DDM analysis. The procedure is based on an optimization cycle (yellow arrows), fed by the experimental image structure function $D(q, t)$ and by an initial set of parameters $\left(A_{0}(q), B_{0}(q)\right)$. The object function is the dispersion $\sigma^{2}$ of the reconstructed mean-square displacements [see Eq. (A3)]. New values of $(A(q), B(q))$ are iteratively generated in order to minimize the object function. The output of the procedure is the optimal set of parameters $(A(q), B(q))$ leading to the best estimate of $\operatorname{MSD}(t)$.

of $[3.02,9.06] \mu \mathrm{m}^{-1}$ for $82.7 \%$ glycerol in water, and of $[3.78,9.06] \mu \mathrm{m}^{-1}$ for $97.5 \%$ glycerol in water. In practice, the width of the wave-vector range is set by the $q$ region in which both $A(q)$ and $B(q)$ are known accurately and Eq. (7) can be used to obtain the MSD from the intermediate scattering functions. For each wave vector in the range [3.78,9.06] $\mu \mathrm{m}^{-1}$ we thus extracted an estimate for the MSD. These estimates were then combined to obtain a $q$-averaged estimate of the MSD for all the samples. These MSDs are reported in Fig. 2(a) for the four Newtonian samples investigated here. All the curves are in excellent agreement with the PT results obtained by analyzing the same image sequences. For the viscosity we obtain $\eta_{\text {meas }}=0.92 \pm 0.03,4.4 \pm 0.2,73 \pm 3,633 \pm$ $40 \mathrm{mPa}$ s. These values are in very good agreement with those expected: $\eta_{\text {lit }}=0.914 \pm 0.01,4.9 \pm 0.05,69.2 \pm 0.7,702 \pm$ $50 \mathrm{mPa} \mathrm{s}$, [45], as shown in the inset of Fig. 2(a). The experimental uncertainty in the value obtained for $97.5 \%$ glycerol in water is due to the uncertainty in the sample composition.

\section{B. Newtonian fluids: Optimization-based differential dynamic microscopy analysis}

As we show in the following, the satisfactory results obtained by using the standard DDM analysis with Newtonian samples depends on the fact that a model for the intermediate scattering function is readily available. In general, this would not be the case, because the behavior of a generic soft material is not known a priori. For this reason, we devised a simple, self-consistent procedure that exploits the multi- $q$ capability of DDM to extract the MSD of tracer particles for an arbitrary sample in a robust way. The proposed procedure builds on the automatic determination of $A(q)$ and $B(q)$ based on an iterated optimization cycle.

The general idea is sketched in Fig. 3, where we show a block diagram that depicts our fitting-free procedure. This procedure is based on an optimization cycle initially fed by a tentative amplitude-baseline parameter pair $\left(A_{0}(q), B_{0}(q)\right)$, for $q$ values within a given interval $\left[q_{1}, q_{2}\right]$. These parameters are used to invert the corresponding image structure functions [Eq. (7)], leading to a "bundle" of MSDs. If the considered pair $\left(A_{0}(q), B_{0}(q)\right)$ is the correct one for all $q$, than the estimates for the MSD given by Eq. (7) are completely $q$ independent, resulting in an almost perfect collapse of all the curves. Any deviation of the parameters from the correct values introduces a $q$-dependent dispersion. In our optimization scheme, the dispersion $\sigma^{2}$ of the curves (see Appendix A for details) plays the role of an objective function: new values of $(A(q), B(q))$ are iteratively generated until a minimum of $\sigma^{2}$ is found. This algorithm, implemented in a custom code developed in MATLAB ${ }^{\circledR}$, was found to rapidly and robustly converge to a minimum for a wide range of wave vectors.

Results obtained for the tracer MSD with this optimizationbased procedure [Fig. 2(b)] are in excellent agreement with those obtained with the fitting-based analysis [Fig. 2(a)] over the whole investigated range of delay times $1.3 \times 10^{-3} \mathrm{~s}<$ $t<10^{2} \mathrm{~s}$, which validates the procedure. Also, we note that the $q$-averaged MSD shown in Fig. 2(b) were obtained by averaging the MSD in the range $1.36 \mu \mathrm{m}^{-1}<q<$ $9.06 \mu \mathrm{m}^{-1}$; this range is wider than that probed with the fitting-based procedure. The usable $q$ range is larger in the optimization-based procedure because the full relaxation of the image structure functions is here not a requirement for the determination of the MSD, since $A(q)$ and $B(q)$ can be obtained self-consistently. Let us stress that the optimization- 

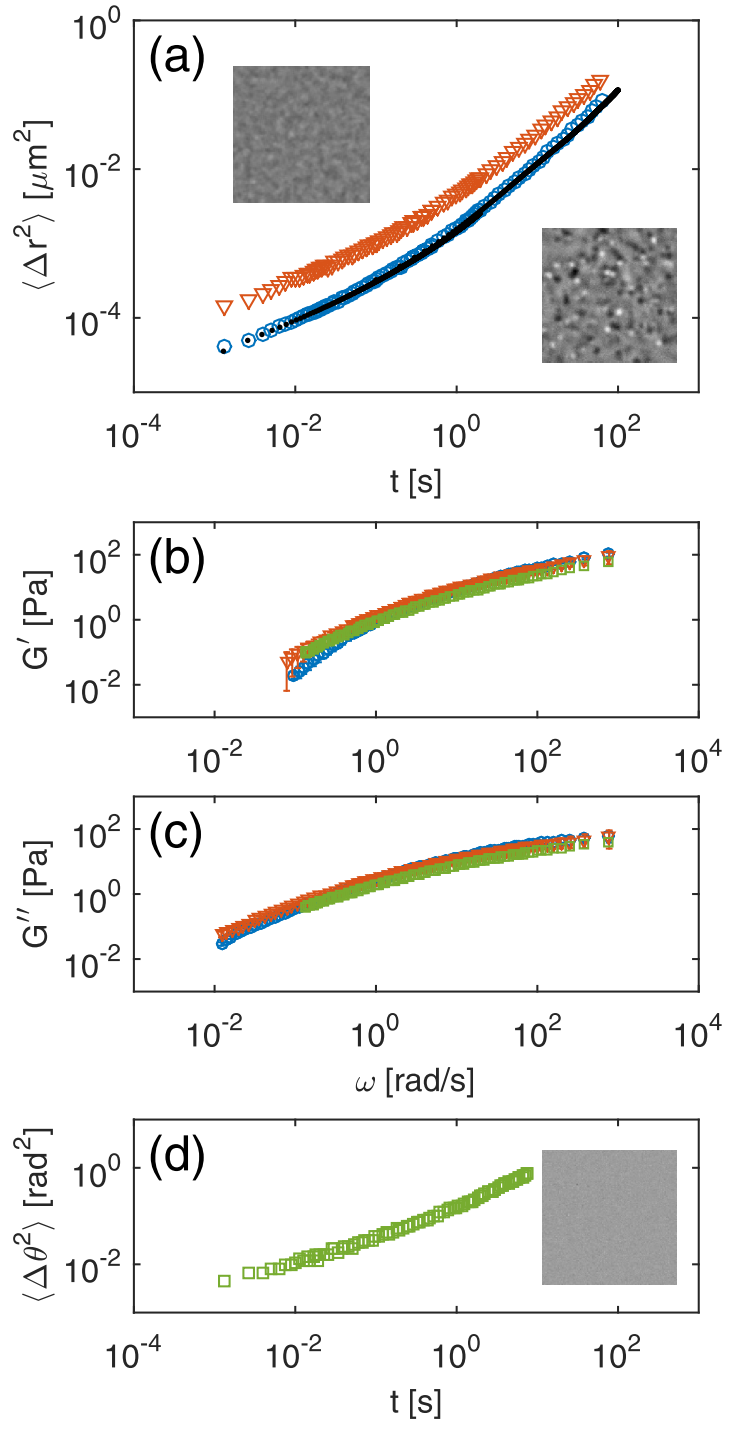

FIG. 4. (a) Two-dimensional MSD of LB1 (orange triangles) and LB5 (blue circles) tracers in a viscoelastic polymer solution (2\% $\mathrm{PEO} 2$ in water) obtained from DDM analysis. Black dots: same quantity obtained from PT analysis for the sample with LB5 tracers. The small insets show representative images of the two samples: the one loaded with subdiffraction LB1 particles (upper-left corner) and the one loaded with LB5 tracers (lower-right corner), respectively. In both cases the image size corresponds to $20.8 \mu \mathrm{m}$. (b) Comparison of the storage moduli $G^{\prime}$ estimated from the DDM-reconstructed MSD of LB1 tracers (orange triangles), LB5 tracers (blue circles), and optically anisotropic tracers (green squares), respectively. (c) Same as in panel (b) for the loss moduli $G^{\prime \prime}$. (d) AMSD of optically anisotropic tracers in a $2 \%$ PEO2 in water solution. The small inset show a representative image of the sample (image size is $83.2 \mu \mathrm{m}$ ).

based procedure is largely model and operator independent. The only required external parameters are the relevant $q$ range $\left[q_{1}, q_{2}\right]$ over which the optimization is performed and the initial values of the parameters $\left(A_{0}(q), B_{0}(q)\right)$. The key importance of all these properties when studying arbitrary samples is described in detail in the next section.

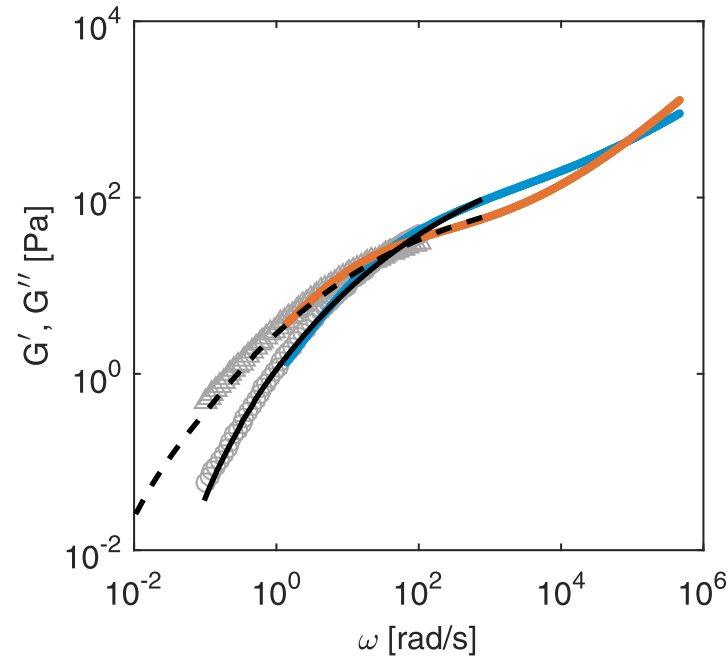

FIG. 5. Comparison of the viscoelastic moduli $G^{\prime}$ and $G^{\prime \prime}$ of a $2 \%$ PEO polymer solution in water, obtained with different methods. Gray circles (triangles): $G^{\prime}\left(G^{\prime \prime}\right)$ obtained with traditional rheology; continuous blue (orange) line: $G^{\prime}\left(G^{\prime \prime}\right)$ obtained with DWS using LB5 tracers; black continuous (dashed) line: $G^{\prime}\left(G^{\prime \prime}\right)$ obtained with DDM microrheology (weighted average of the results of LB1 and LB5 tracers, shown individually in Fig. 4).

\section{Viscoelastic fluid}

In this section, we apply the optimization-based procedure to the data obtained with our model viscoelastic fluid, an aqueous solution of PEO, which exhibits elastic behavior at short times and high frequencies. The expected short-time elastic plateau in the MSD would contribute to the baseline $B(q)$, requiring an independent determination of the camera noise. Such a requirement would be similarly involved as the calibration procedure needed in PT- $\mu$ r experiments to account for the tracer localization uncertainty. While such calibration is technically feasible, the optimization-based DDM analysis permits a calibration-free implementation of DDM- $\mu$ r.

Application of the optimization-based procedure to the PEO solutions with small $(\sim 100 \mathrm{~nm})$ and large $(\sim 500 \mathrm{~nm})$ tracers provides the results shown in Fig. 4(a). The accessible range of timescales probed is very similar for the two tracer sizes. For comparison we also show the results obtained with PT for the sample containing the larger tracers as black points in Fig. 4(a); for the smaller particles tracking is not feasible, as easily appreciated from the images shown as insets.

For each tracer size, we extracted from the MSD the mechanical moduli $G^{\prime}$ and $G^{\prime \prime}$, as shown in Figs. 4(b) and 4(c). Results obtained for $G^{\prime}$ with the two tracer sizes are off by about $10 \%-20 \%$ at small frequencies but the two datasets are compatible within the experimental errors. We combine the data obtained with the two tracers and show the results as black lines in Fig. 5. These data are in good agreement with the results obtained with traditional rheology, shown as open symbols, and also with the results obtained with DWS, shown as closed symbols. DDM- $\mu \mathrm{r}$ extends traditional rheology by one decade at high frequency, whereas at low frequency similar performances are obtained, at least as far as the storage modulus is concerned. However, 

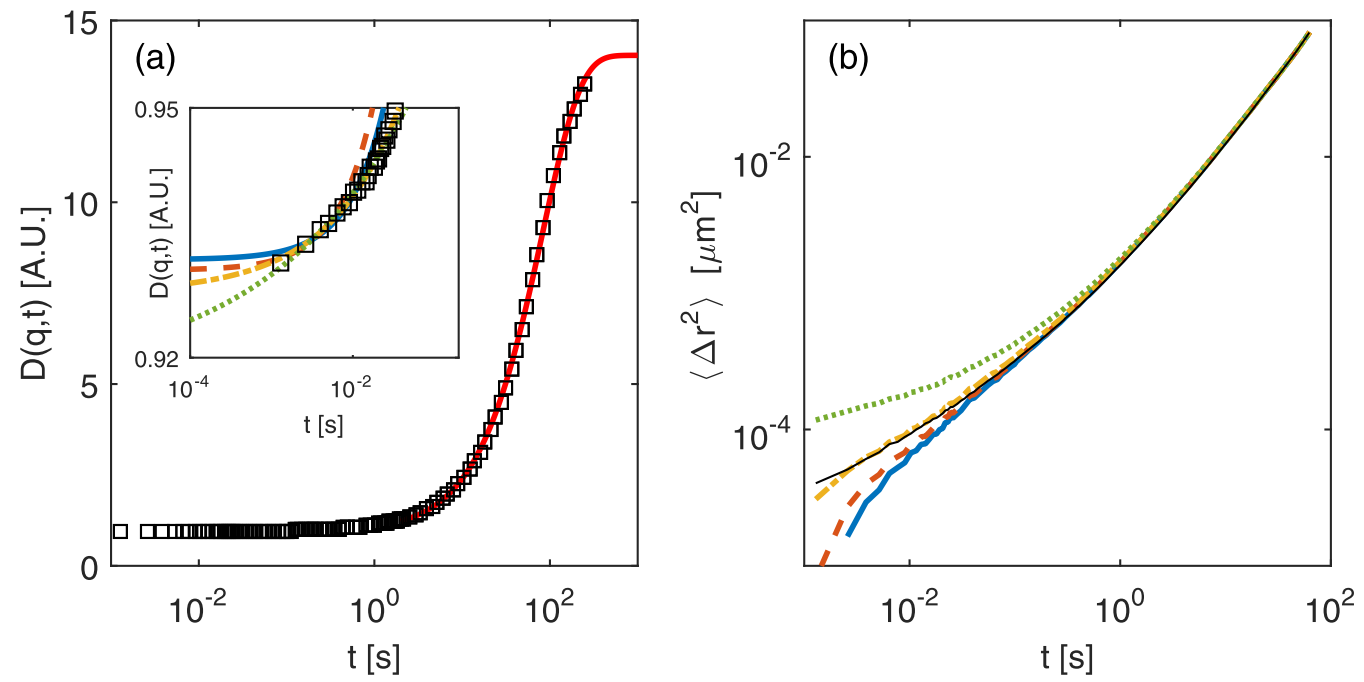

FIG. 6. Effect of the model-dependent determination of the noise baseline $B(q)$ on the reconstructed MSD. (a) Symbols: image structure function $D(q, t)$ (for $q=6.04 \mu \mathrm{m}^{-1}$ ) obtained from DDM on LB5 tracers in a viscoelastic solution of PEO in water. The continuous red line is an exponential fit to the data obtained at large delay times $(t>20 \mathrm{~s})$; this fit allows for the estimate of the plateau height $A^{\prime}(q)$. Inset shows a close-up of the short time behavior of $D(q, t)$ (symbols). The data are fit with different functions, leading to different estimates of the baseline $B(q)$ : linear fit over the first 10 data points (continuous blue line), linear fit over the first 5 data points (dashed orange line), fit over the first 20 data points with a function of the form $y=a x^{0.5}+b$ (dashed-dotted yellow line), and fit over the same interval with a function of the form $y=a x^{0.25}+b$ (dotted green line). (b) Mean-square displacement obtained from Eq. (6) by using the amplitude $A(q)=A^{\prime}(q)-B(q)$ and the noise baseline $B(q)$ obtained from the different fitting models shown in the inset of panel (a). Curves are color coded according to the fits, the black continuous line is the result of the model-free procedure shown in Fig. 4(a) as blue circles.

improvements in the low-frequency region may be expected by increasing the mechanical stability of the microscope setup.
Let us underline that, without additional calibration steps, it would be very difficult to extract meaningful MSD and thus mechanical moduli with a fitting-based analysis of the DDM
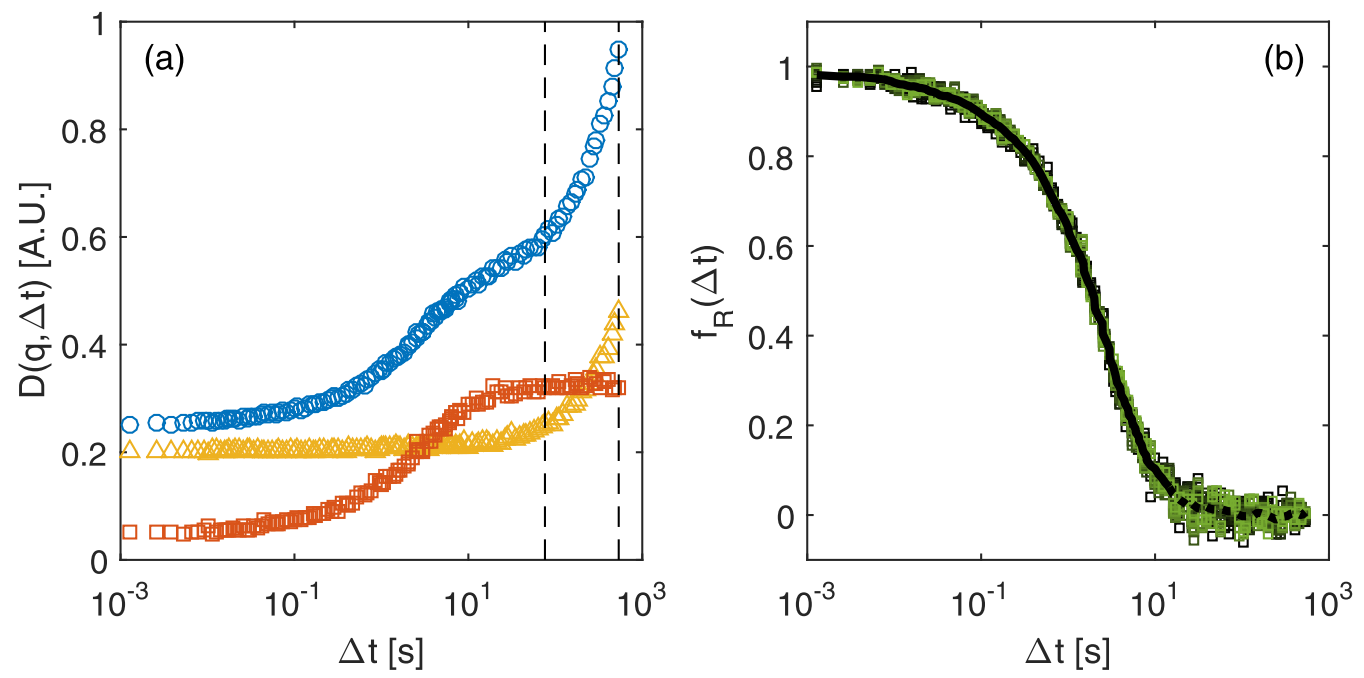

FIG. 7. Isolation of the rotational dynamics from the combination of bright-field and polarized microscopy measurements. (a) Yellow upward triangles: image structure function $D(q, t)$ (for $q=0.94 \mu \mathrm{m}^{-1}$ ) obtained from bright-field DDM on optically anisotropic tracers in a viscoelastic solution of PEO in water. The curve shows a single decay, associated with the translational dynamics of the tracers. Blue circles: image structure function $D_{p}(q, t)\left(q=0.94 \mu \mathrm{m}^{-1}\right.$, as before $)$ obtained from polarized DDM on the same sample, showing two distinct decays: a fast one, associated with the roto-translational dynamics of the tracers, and a slower one due to their translational dynamics alone. A suitably weighted difference of $D_{P}$ and $D$ (see main text and Appendix B for details on the optimization procedure used for the calculation of the weighting factor) leads to the curve represented by orange squares, accounting for roto-translational dynamics alone. The vertical dashed lines enclose the time interval where the optimization procedure is performed. (b) Symbols: overplot of all the intermediate scattering functions obtained from the procedure illustrated in panel (a) for $0.038 \mu \mathrm{m}^{-1}<q<1.0 \mu \mathrm{m}^{-1}$. The black curve is an average of the intermediate scattering functions obtained at different wave vectors. The portion of the curve shown as a continuous line (corresponding to $f_{R}(t)>0.05$, or $1.3 \times 10^{-3} \mathrm{~s}<t<1.0 \mathrm{~s}$ ) is the one used to extract the AMDS shown in Fig. 4(d). 
data. In the limit of short times the MSD displays a nontrivial scaling, compatible with a power law $\mathrm{MSD} \simeq t^{\gamma}$ with an exponent $\gamma$ close to 0.5 . The counterpart of this behavior in the Fourier space is an image structure function taking the form of $D(q, t) \simeq C(q) t^{\gamma}+B(q)$ at short times. Clearly, an exponential or a polynomial fit of $D(q, t)$ is inadequate to describe this behavior and any estimate of the baseline $B(q)$ based on an exponential or a polynomial fit provides a biased, incorrect result, as shown in Fig. 6. Choosing other model functions, such as, for instance, a power law with different fixed exponents, also fails, even though the data may seem deceivingly well described at short times. By contrast, the optimization-based procedure self-consistently determines the MSD without need of fitting the experimentally determined image structure functions.

After seeding with optically anisotropic tracers, we measured the same PEO solution with both standard and polarized DDM, according to the procedure described in Sec. II F and in Appendix B. The obtained AMSD is shown in Fig. 4(d). As can be appreciated from the small inset showing a typical experimental image after subtraction of the static background, the optical contrast of the subdiffraction birefringent particles was extremely low. This fact, combined with the intrinsic fluctuation in the particle's intensity due to their rotational dynamics, made PT absolutely impossible in this case. By comparing Figs. 4(a) and 4(d), we note that the time interval over which the AMSD can be accurately extracted is more limited compared with the translational MSD. This is due to the fact that, after a delay time of approximately $t \simeq 1.0 \mathrm{~s}$, the orientation of the particles decorrelates almost completely $\left[f_{R}(t)<0.05\right.$, see also Fig. 7(b)] and the inversion described by Eq. (10) becomes unreliable. The corresponding estimates for $G^{\prime}$ and $G^{\prime \prime}$ (green symbols in Fig. 4) obtained from Eq. (2) are in remarkably good agreement with the results obtained with standard DDM, even though they are affected by a $\simeq 50 \%$ uncertainty that is mainly due to the $\simeq 15 \%$ uncertainty in the particle size.

\section{CONCLUSIONS}

Microrheology is a very powerful complement to traditional, mechanical rheology [5,7-9]. For the high-frequency range, rheology is usefully complemented by DWS- $\mu$ r [10], whereas in the low-frequency limit both DLS- $\mu \mathrm{r}$ [11] and PT- $\mu$ r [6] have been usefully employed in the past. PT- $\mu \mathrm{r}$ is technically the less demanding technique, not requiring any laser source or digital correlation board and is also very flexible for biophysical applications, owing to the possibility of employing different sample contrast mechanisms. However, in its practical realization one encounters some challenges. Accurate tracking algorithms require several input parameters, such as a typical value for the particle radius, a score cutoff to discriminate signals that are not due to the presence of particles, an intensity threshold to consider bright pixels as particles, etc. The results of the tracking depends severely on the choice of these parameters that, even for experienced users, may be sometimes more difficult than expected [48]. Also, the extraction of the tracer MSD from PT trajectories requires the knowledge of the intrinsic particle localization uncertainty, which is usually determined by calibration with particles that are kept fixed in space or that freely diffuse in a Newtonian fluid with similar optical properties [8].

We have shown here that DDM [21], a technique that retains the simplicity and flexibility of PT in terms of experimental setup and applications, can be also used for accurate microrheology experiments probing both the translational and the rotational degrees of freedom of embedded tracer particles. We also show that PT is outperformed by DDM in bright-field DDM experiments probing translational dynamics as well as in p-DDM experiments probing rotational motion. Finally, if an optimization-based algorithm is used instead of the standard fitting-based approach, DDM- $\mu$ r does not require any calibration or user input, which limits dramatically the degree of arbitrariness on the determination of the mechanical moduli of the sample. However, particle tracking is expected to be superior to DDM in the presence of unwanted and moving scatterers that, being potentially discarded by an accurate particle tracking, would affect DDM- $\mu$ r experiments.

It is likely that these and other DDM features, such as its capability to handle optically dense samples, for which tracking becomes extremely challenging if not impossible, will make DDM- $\mu \mathrm{r}$ a useful addition to the portfolio of rheo-scientists, both in academic and in industrial research laboratories.

Future developments might include the study of materials that are nonhomogeneous at the scale of the probe particles, or situations in which the probe particles are interacting or polydisperse. In these cases, the GSERs and Eq. (6) would be not valid, and one would need to replace them by appropriate relations. Finally, particularly intriguing appears the possibility of probing the microrheological properties of materials without added tracers, i.e., by exploiting the scattering of light from the material constituents themselves.

\section{ACKNOWLEDGMENTS}

We thank Elisa Tamborini, Giuliano Zanchetta, Stefano Buzzaccaro, and Marco Caggioni for stimulating discussions. We thank Catalina Haro-Pérez for the gift of the optically anisotropic particles. We thank Gora Conley for careful reading of the manuscript and Marco Ciccarelli for help with the p-DDM experiments. We acknowledge funding from the Italian Ministry of University and Scientific Research (MIUR) - Project RBFR125H0M, from Regione Lombardia and CARIPLO foundation - Project 2016-0998 and from the Swiss National Science Foundation - Project 200021-157214.

\section{APPENDIX A: OPTIMIZATION-BASED DETERMINATION OF MEAN-SQUARE DISPLACEMENT}

In this Appendix, we describe the fitting-free optimization procedure used to extract from the experimental image structure function $D(q, t)$ the best estimate for the tracers' mean-square displacement. The main steps of the procedure are the following:

(1) Choice of the interval $\left[q_{1}, q_{2}\right]$ of wave vectors over which the optimization is performed. The interval should be a subset of the accessible $q$ range with a fair signal-to-noise ratio. This condition can be also checked retrospectively at 
the end of the procedure, when a $q$ resolved estimate of the amplitude $A(q)$ and the noise background $B(q)$ is obtained.

(2) Choice of the initial set of parameters $\left(A_{0}(q), B_{0}(q)\right)$. This can be done, for example, by fitting, for each $q \in\left[q_{1}, q_{2}\right]$, $D(q, t)$ with a linear function near the origin and with a exponential function for large delays (as done, for example, in Fig. 6).

(3) Calculation of the mean-square displacement $\operatorname{MSD}(t \mid q)$ using Eq. (6) for each $q$ in the selected interval.

(4) Determination, for each delay time $t$, of the subset $J(t)$ of $q$ values such that $\operatorname{MSD}(t \mid q)<q^{-2}$. This choice ensures that, if $q \in J(t)$, then $D(q, t)$ has not completely lost track of the signal correlation for that value of $q$ and can thus be meaningfully inverted. Let $N(t)$ be the number of elements in $J(t)$.

(5) Calculation of the average mean-square displacement

$$
\operatorname{MSD}(t)=\frac{1}{N(t)} \sum_{q \in J(t)} \operatorname{MSD}(t \mid q) .
$$

(6) Calculation of the $t$-dependent dispersion $\sigma_{t}^{2}(t)$ as

$$
\sigma_{t}^{2}(t)=\frac{1}{N(t)-1} \sum_{q \in J(t)} \log ^{2} \frac{\operatorname{MSD}(t \mid q)}{\operatorname{MSD}(t)}
$$

and of the total dispersion $\sigma^{2}$ as

$$
\sigma^{2}=\sum_{t} \sigma^{2}(t)
$$

(7) Generation of a new set of parameters and repetition of the procedure from step 3 unless a local minimum in $\sigma^{2}$ is reached (or the prescribed maximum number of iterations is exceeded).

(8) If the procedure converges to a minimum of $\sigma^{2}$, the optimal set of parameters $(A(q), B(q))$ represents the best estimate for the $q$-dependent amplitude and noise baseline, respectively, and the corresponding average mean-square displacement [Eq. (A1)] is the best estimate for the tracer's MSD.

Many algorithms are available to search for the minimum of $\sigma^{2}$ and to guide the generation of new sets of parameters in step 7. In our implementation, the optimization cycle 3-7 was realized by using the MATLAB function fminsearch, which is based on the simplex search method of Lagarias et al. [49]. A more refined implementation could possibly include suitable weights when computing the averages in the right-hand sides of Eqs. (A1)-(A3), accounting for the different statistical errors affecting each term. Also, an effective weighting scheme could provide an efficient way to reject the contribution of the most noisy wave vectors making unnecessary the explicit selection of a predetermined optimization interval (step 1).

\section{APPENDIX B: DETERMINATION OF THE AMSD FROM COMBINED p-DDM AND BRIGHT-FIELD DDM MEASUREMENTS}

In this Appendix, we describe the procedure used to extract the angular mean-square displacement from the experimental image structure functions $D_{p}$ and $D$ determined, for the same sample seeded with optically anisotropic tracers, with p-DDM and bright-field DDM, respectively. Inspection of Eqs. (5), (6), (8), and (9) reveals that the translational intermediate scattering function $f_{T}$ contributes additively to both $D$ and $D_{p}$. This suggests that its contribution could be eliminated-and thus the roto-translational dynamics (encoded in $f_{R T}$ ) isolated-by considering a suitably weighted difference of $D_{p}$ and $D$

$$
D_{d}(q, t)=D_{p}(q, t)-c(q) D(q, t) .
$$

According to Eqs. (5), (6), (8), and (9) we can remove any additive contribution of the translational intermediate scattering function by choosing the weighting factor $c(q)=$ $[1-\alpha(q)] \frac{A_{p}(q)}{A(q)}$. We instead determined $c(q)$ directly for each $q$ as the coefficient that, by canceling out the translational decay, minimizes the slope of $D_{d}(q, t)$ over a suitably chosen time interval $\left[t_{1}, t_{2}\right]$ [Fig. 7(a)]. The lower endpoint $t_{1}$ is taken large enough to ensure the almost complete decorrelation of the roto-translational term $f_{R T}\left(t_{1}\right) \simeq 0$, while the upper endpoint $t_{2}$ is chosen as the largest time delay for which the image structure function could be accurately sampled. In our experimental condition, we set $t_{1}=70 \mathrm{~s}$ and $t_{2}=700 \mathrm{~s}$. The output of this procedure is a collection of corrected image structure functions

$$
D_{d}(q, t)=A_{d}(q)\left[1-f_{R T}(q, t)\right]+B_{d}(q)
$$

showing, for each $q$, a single decay associated with the rototranslational dynamics of the tracers. Qualitatively, if $q$ is low enough, the translational dynamics is expected to be much slower than the rotational one and $f_{R T}$ almost $q$ independent and indistinguishable from $f_{R}(t)$. This argument can be made more rigorous by noticing that

$$
\ln f_{R T}(q, t)=-3\left\langle\Delta \theta^{2}(t)\right\rangle[1+\epsilon(q)],
$$

where

$$
\epsilon(q)=\frac{\ln f_{T}(q, t)}{\ln f_{R}(t)}=\frac{q^{2}\left\langle\Delta r^{2}(t)\right\rangle}{12\left\langle\Delta \theta^{2}(t)\right\rangle}=\frac{2}{9}(a q)^{2},
$$

and the last equality is a direct consequence of Eqs. (1) and (2). Equations (B3) and (B4) show that the error done in estimating $\left\langle\Delta \theta^{2}(t)\right\rangle$ directly as $-\frac{1}{3} \ln f_{R T}$ is of the order $(a q)^{2}$. In particular, since in our case $a \simeq 0.1 \mu \mathrm{m}$, if we restrict ourselves to $q<1 \mu \mathrm{m}^{-1}$ [where $\epsilon(q)<3 \times 10^{-3}$ ], we can write, within a $1 \%$ accuracy:

$$
\left\langle\Delta \theta^{2}(t)\right\rangle \simeq-\frac{1}{3} \ln f_{R T}(q, t) .
$$

The robustness of this approximation can be visually checked in Fig. 7(b), where the intermediate scattering functions extracted for different $q$ values comprised in the range $[0.038,1] \mu \mathrm{m}^{-1}$ show a nice collapse on a single curve.

Building on this result, we average all the corrected image structure functions in the specified $q$ range, obtaining

$$
\bar{D}_{d}(t)=\sum_{q} D_{d}(q, t) \simeq a\left[1-f_{R}(t)\right]+b .
$$

Finally, in analogy with Eq. (7), we extract the AMSD as

$$
\left\langle\Delta \theta^{2}(t)\right\rangle=-\frac{1}{3} \ln \left(1-\frac{\bar{D}_{d}(t)-b}{a}\right) .
$$


Here the baseline $b$ is determined by fitting the short-time behavior of $\bar{D}_{d}(t)$ to a function of the form $c t^{\gamma}+b$, while the amplitude $a$ is robustly estimated as the average of $\bar{D}_{d}(t)$ over the interval $\left[t_{1}, t_{2}\right]$.
[1] P. Coussot, Rheophysics: Matter in all its States (Springer, Cham, 2014).

[2] H. M. Wyss, Rheology of soft materials, in Fluids, Colloids and Soft Materials (John Wiley and Sons, Hoboken, New Jersey, 2016), pp. 149-163.

[3] G. M. Kavanagh and S. B. Ross-Murphy, Prog. Polym. Sci. 23, 533 (1998)

[4] R. H. Ewoldt, M. T. Johnston, and L. M. Caretta, Experimental challenges of shear rheology: How to avoid bad data, in Complex Fluids in Biological Systems (Springer, New York, 2015), pp. 207-241.

[5] P. Cicuta and A. M. Donald, Soft Matter 3, 1449 (2007).

[6] T. G. Mason, K. Ganesan, J. H. van Zanten, D. Wirtz, and S. C. Kuo, Phys. Rev. Lett. 79, 3282 (1997).

[7] T. M. Squires and T. G. Mason, Annu. Rev. Fluid Mech. 42, 413 (2010).

[8] T. A. Waigh, Rep. Prog. Phys. 68, 685 (2005).

[9] T. A. Waigh, Rep. Prog. Phys. 79, 074601 (2016).

[10] T. G. Mason and D. A. Weitz, Phys. Rev. Lett. 74, 1250 (1995).

[11] T. Mason, H. Gang, and D. Weitz, J. Mol. Struct. 383, 81 (1996).

[12] B. R. Dasgupta, S. Y. Tee, J. C. Crocker, B. J. Frisken, and D. A. Weitz, Phys. Rev. E 65, 051505 (2002).

[13] S. Rathgeber, H.-J. Beauvisage, H. Chevreau, N. Willenbacher, and C. Oelschlaeger, Langmuir 25, 6368 (2009).

[14] Z. Cheng and T. G. Mason, Phys. Rev. Lett. 90, 018304 (2003).

[15] R. Colin, L. Chevry, J.-F. Berret, and B. Abou, Soft Matter 10, 1167 (2014).

[16] R. Cerbino, D. Piotti, M. Buscaglia, and F. Giavazzi, J. Phys.: Condens. Matter 30, 025901 (2018).

[17] E. Andablo-Reyes, P. Díaz-Leyva, and J. L. Arauz-Lara, Phys. Rev. Lett. 94, 106001 (2005).

[18] C. Haro-Pérez, E. Andablo-Reyes, P. Díaz-Leyva, and J. L. Arauz-Lara, Phys. Rev. E 75, 041505 (2007).

[19] M. A. Escobedo-Sánchez, A. Hugo, J. L. Arauz-Lara, C. Haro-Pérez, and L. F. Rojas-Ochoa, J. Chem. Phys. 143, 044902 (2015).

[20] F. Giavazzi, C. Haro-Pérez, and R. Cerbino, J. Phys.: Condens. Matter 28, 195201 (2016).

[21] R. Cerbino and V. Trappe, Phys. Rev. Lett. 100, 188102 (2008).

[22] F. Croccolo, D. Brogioli, A. Vailati, M. Giglio, D. S. Cannell, and S. S. Sadhal, Ann. NY Acad. Sci. 1077, 365 (2006).

[23] F. Giavazzi and R. Cerbino, J. Opt. (Bristol, UK) 16, 083001 (2014).

[24] F. Giavazzi, D. Brogioli, V. Trappe, T. Bellini, and R. Cerbino, Phys. Rev. E 80, 031403 (2009).

[25] L. G. Wilson, V. A. Martinez, J. Schwarz-Linek, J. Tailleur, G. Bryant, P. N. Pusey, and W. C. K. Poon, Phys. Rev. Lett. 106, 018101 (2011).
[26] M. Reufer, V. A. Martinez, P. Schurtenberger, and W. C. K Poon, Langmuir 28, 4618 (2012).

[27] K. He, M. Spannuth, J. C. Conrad, and R. Krishnamoorti, Soft Matter 8, 11933 (2012).

[28] Y. Gao, J. Kim, and M. E. Helgeson, Soft Matter 11, 6360 (2015).

[29] D. Germain, M. Leocmach, and T. Gibaud, Am. J. Phys. 84, 202 (2016).

[30] A. Dehaoui, B. Issenmann, and F. Caupin, Proc. Natl. Acad. Sci. USA 112, 12020 (2015).

[31] A. Wittmeier, A. Leeth Holterhoff, J. Johnson, and J. G. Gibbs, Langmuir 31, 10402 (2015).

[32] T. Sentjabrskaja, E. Zaccarelli, C. De Michele, F. Sciortino, P. Tartaglia, T. Voigtmann, S. U. Egelhaaf, and M. Laurati, Nat. Commun. 7, 11133 (2016).

[33] D. M. Wulstein, K. E. Regan, R. M. Robertson-Anderson, and R. McGorty, Opt. Express 24, 20881 (2016).

[34] T. E. Kodger, P. J. Lu, G. R. Wiseman, and D. A. Weitz, Langmuir 33, 6382 (2017).

[35] R. Cerbino and P. Cicuta, J. Chem. Phys. 147, 110901 (2017).

[36] A. V. Bayles, T. M. Squires, and M. E. Helgeson, Soft Matter 12, 2440 (2016).

[37] A. V. Bayles, T. M. Squires, and M. E. Helgeson, Rheol. Acta 56, 863 (2017).

[38] M. Drechsler, F. Giavazzi, R. Cerbino, and I. M. Palacios, Nat. Commun. 8, 1520 (2017).

[39] A. Mertelj, J. L. Arauz-Lara, G. Maret, T. Gisler, and H. Stark, Europhys. Lett. 59, 337 (2002).

[40] P. Díaz-Leyva, E. Pérez, and J. L. Arauz-Lara, J. Chem. Phys. 121, 9103 (2004).

[41] J. Bibette, J. Colloid Interface Sci. 147, 474 (1991).

[42] http://people.umass.edu/kilfoil.

[43] J. C. Crocker and D. G. Grier, J. Colloid Interface Sci. 179, 298 (1996).

[44] D. A. Weitz and D. J. Pine, Diffusing-wave spectroscopy, in Dynamic Light Scattering: The Method and Some Applications (Clarendon Press, New York, 1993), pp. 652-720.

[45] N.-S. Cheng, Ind. Eng. Chem. Res. 47, 3285 (2008).

[46] F. Giavazzi, P. Edera, P. J. Lu, and R. Cerbino, Eur. Phys. J. E: Soft Matter Biol. Phys. 40, 97 (2017).

[47] B. J. Berne and R. Pecora, Dynamic Light Scattering: With Applications to Chemistry, Biology, and Physics (Dover Publications, New York, 2000).

[48] N. Chenouard, I. Smal, F. De Chaumont, M. Maška, I. F. Sbalzarini, Y. Gong, J. Cardinale, C. Carthel, S. Coraluppi, M. Winter et al., Nat. Methods 11, 281 (2014).

[49] J. C. Lagarias, J. A. Reeds, M. H. Wright, and P. E. Wright, SIAM J. Optimiz. 9, 112 (1998). 